SYPHILIS

\title{
Lessons from the syphilis outbreak in homosexual men in east London
}

\author{
M Hourihan, H Wheeler, R Houghton, B T Goh
}

Sex Transm Infect 2004;80:509-511. doi: 10.1136/sti.2004.011023

Objectives: To describe the epidemiology, presentation, and diagnosis of early syphilis in 103 homosexual men in east London.

Methods: A retrospective study using data from KC60 returns, the Health Protection Agency (HPA) enhanced surveillance forms and case notes.

Results: 40 cases of primary (PS), 40 of secondary (SS) and 23 of early latent syphilis were identified, 33\% co-infected with HIV. $41 \%$ had concurrent sexually transmitted infections (STIs). Pain featured in 35\% of PS and itch in 13\% of rashes. Dark ground microscopy (DGM), performed in 44 of the symptomatic cases, was positive in $37(84 \%)$ allowing early management. Initial syphilis serology was negative in 15/40 (37\%) cases of PS. $51 \%$ and $49 \%$ opted for parenteral and oral treatment, respectively. In 53/103 (51\%) cases oral sex was the only risk factor. $86 \%$ of infections were UK acquired. Only $4 \%$ of contacts were seen.

Conclusion: This outbreak, reflecting the resurgence of syphilis across the United Kingdom, highlights several important points. Painful chancres and itchy rash are common presentations. DGM is a highly sensitive diagnostic tool. Initial negative serological screening tests are common in PS and sero-surveillance for 3 months is recommended. The high prevalence of concomitant STls indicates ongoing unprotected sexual intercourse. Oral sex is a significant risk factor and is a distinctly "unsafe" practice. Conventional partner notification is ineffective. Other methods of screening of the at-risk homosexual population are warranted. Continued education is required to reduce STI acquisition in homosexual men.

O utbreaks of infectious syphilis reported in the United Kingdom, primarily in homosexual men, have led to a $250 \%$ increase in its incidence over the past 5 years. ${ }^{1-6}$ Untreated syphilis has recognised complications and, being an ulcerative disease, may facilitate the transmission of other STIs including HIV. HIV may also modify the manifestations of syphilis. ${ }^{78}$ Therefore, it is essential that prompt diagnosis, treatment, and contact tracing occur in order to control this major outbreak. We describe the epidemiology, presentation, and diagnosis of early syphilis in homosexual men who attended two east London clinics.

\section{METHODS}

A retrospective notes review of homosexual men diagnosed with early syphilis between April 2001 and December 2002, identified using KC60 returns and HPA enhanced syphilis surveillance forms was performed. Demographics were recorded with details of presentation, results of diagnostic tests, concurrent infections and treatments, likely route and place of acquisition, and details of contact tracing.

\section{RESULTS}

A total of 103 cases were identified, 40 with primary (PS), 40 with secondary (SS) and 23 with early latent syphilis. The median age was 36 years (range 17-56) and 93\% were white, 5\% Asian and 2\% Afro-Caribbean.

\section{Clinical presentation}

The lesions of PS were located on the penis (27), anal margin (eight), and mouth (five). Chancres were painful in 14 (35\%) cases, six were initially treated as for ano-genital herpes; one patient was both HSV culture and DGM positive. Men with SS presented with rash alone (25), rash and muco-cutaneous ulcers (nine), rash and generalised lymph nodes (four), rash, lymph nodes, and ulcers (two). In $10(25 \%)$ cases of SS, the primary lesion was still present. The rash was itchy in five cases, being the predominant symptom in these men. HIV positive patients presented as PS (15\%), SS (59\%), and early latent syphilis $(26 \%)$.

\section{Concurrent STIs}

In all, 34\% of the cohort were known to be HIV positive, 34\% tested negative, while the others declined testing. Of 99 accepting a sexual health screen, in 43 co-existing STIs were diagnosed, three patients having more than one STI. Of eight with rectal gonorrhoea, six were HIV positive (table 1).

\section{Diagnostics}

Of 40 with PS, 27 had dark ground microscopy (DGM) performed (no intra-oral lesions) of which 22 (81\%) were positive. Fifteen of $40(38 \%)$ cases had negative initial syphilis serology (treponemal EIA- murex ICEsyphilis), 14 of which were sent to the HPA Reference Laboratory, Bristol and were all found to be EIA IgM positive. All 40 cases of SS had positive initial EIA. DGM was performed in 17 cases and this was positive in $15(88 \%)$. The main reasons why DGM was not performed were equipment failure, trained staff unavailability, or ulcers were too painful and were initially diagnosed as ano-genital herpes.

\section{Route of acquisition and partner notification}

Fifty four patients $(52 \%)$ stated that the only risk factor was unprotected oral intercourse (UPOI). Of the 35 HIV positive patients, 57\% were known to have a detectable plasma viral load. Nineteen of 35 patients (54\%) reported unprotected anal intercourse (UPAI). Most men (86\%) reported likely acquisition in the United Kingdom. Of a total of 1279 contacts cited, only 122 (10\%) were known and contactable, with $43(4 \%)$ being successfully traced and screened. Social networks identified by the 103 index cases were bars $(54 \%)$,

Abbreviations: DGM, dark ground microscopy; GUD, genital ulcerative disease; HPA, Health Protection Agency, PS, primary syphilis; pVL, plasma HIV-RNA viral load; SS secondary syphilis, ; STI, sexually transmitted infections; UPAl, unprotected anal intercourse; UPOI, unprotected oral intercourse 
Table 1 Concurrent sexually transmitted infections identified

\begin{tabular}{ll}
\hline STI & Number \\
\hline Gonorrhoea & 17 (8 rectal) \\
Non-gonococcal urethritis & 9 \\
Chlamydial infection & 4 \\
Ano-genital warts & 9 \\
Ano-genital herpes & 2 \\
Acute hepatitis B & 1 \\
HIV infection & 1 \\
\hline
\end{tabular}

sauna $(9 \%)$, internet $(9 \%)$, public sex venues $(5 \%)$, and escorts $(2 \%)$.

\section{Treatment}

In all, $51(49.5 \%)$ received parenteral treatment and 51 oral doxycycline $100 \mathrm{mg}$ twice daily for 2 weeks or $200 \mathrm{mg}$ twice daily for 4 weeks. Forty five patients received procaine penicillin intramuscularly $600 \mathrm{mg}$ once daily for 10 days or $1.8 \mathrm{~g}$ once daily with oral probenecid $500 \mathrm{mg}$ four times daily for 17 days; six had benzathine penicillin intramuscularly 2.4 megaunits weekly for two injections. One received oral amoxycillin $2 \mathrm{~g} /$ probenecid $500 \mathrm{mg}$ four times daily for 28 days. In known HIV patients, regimens were as for neurosyphilis. ${ }^{9}$

\section{DISCUSSION}

The lessons from this case study are distinctive and provide a greater understanding of this current outbreak.

"Classic" presentations such as painless chancres and nonitchy rash should not be perpetuated. Genital ulcers were painful in 35\%, imitating ano-genital herpes. The rash of SS may be itchy and this was a feature in $13 \%$ in this study of predominantly white men. In another study, $10 \%$ had an itchy rash but $40 \%$ of patients were black. ${ }^{10}$ This may cause diagnostic confusion with other aetiologies such as drug rash.

Any homosexual man with genital ulcerative disease (GUD) should have DGM performed. This has been shown to be highly sensitive in trained hands, allowing early intervention. ${ }^{11}$ The lack of sensitivity of the screening syphilis serology in PS emphasises the need for DGM and serosurveillance for up to 3 months in undiagnosed GUD. ${ }^{11-14}$

Almost equal numbers opt for parenteral or oral treatment; the popularity of the latter is based on convenience; encouragingly, all who opted for daily procaine penicillin injections completed their course. The long term efficacy of oral treatment is unknown and requires clinical and serological follow up, particularly in HIV cases, as clinical relapse is a real possibility in this group.

Fifty one per cent of patients stated oral sex as the only risk factor. While this is perceived as low risk for acquisition of HIV, it is considered high risk for the transmission of syphilis as oral lesions are highly infectious. The high rates of concurrent STIs found, including rectal gonorrhoea, suggest ongoing UPAI. Worryingly, there was a high rate of UPAI reported among HIV positive cases; $57 \%$ of the co-infected patients had detectable plasma HIV-RNA viral load ( $p V L)$. The presence of syphilitic lesions could result in increased transmission of HIV as inflammation, secondary to infection, increases local HIV RNA, and raised pVL correlates with higher semen HIV-RNA. ${ }^{15}$ Not infrequently, HIV infected patients present with more severe GUD, neurosyphilis, and other complications. ${ }^{78}$ This, together with the high prevalence of STIs in HIV patients, supports the recommendation that HIV patients should receive comprehensive sexual health care integrated with their specialist care. ${ }^{16}$ Syphilis screening at 3 monthly intervals, if HIV positive, has been shown to yield significant numbers of asymptomatic cases. ${ }^{17}$

Non-effective partner notification is a concern, with reported incidence of early syphilis in contacts of $26 \%{ }^{18}$ and $21 \% .^{19}$ Interventions to case find and impact on the present outbreak are difficult as the majority of partners were casual and untraceable, as they were met through bars, saunas, internet, and public sex venues. Some identifiable contacts may have accessed other clinics. The Terence Higgins Trust has a campaign to promote awareness of the syphilis outbreak in gay venues ${ }^{20}$ but its efficacy is unclear. The value of case finding using treponemal serology at homosexual venues is also uncertain. ${ }^{21}$ The development of an oral test or real time desktop rapid blood tests, providing immediate diagnosis, are more user friendly and may prove more useful than current serology for case finding. ${ }^{23}$ There is a suggestion that sexual health promotion through the internet may be feasible. ${ }^{24}$ Other strategies will need to be developed to control the current outbreak of syphilis in the United Kingdom.

A limitation of this study is that it is retrospective. A prospective qualitative study looking at disease presentation and assessing partner non-notification will provide further understanding of issues and enable appropriate and gender sensitive interventions.

\section{ACKNOWLEDGEMENTS}

The authors would like to thank the administrative, nursing, and health advising staff of Barts Sexual Health Centre and the Ambrose King Centre.

\section{CONTRIBUTORS}

$\mathrm{MH}$ and HW participated in the protocol development, collected the data and are the principal authors; RH assisted with data collection; BTG initiated the study, developed the protocol, and critically revised the paper.

\section{Authors' affiliations}

M Hourihan, H Wheeler, R Houghton, B T Goh, Ambrose King Centre, Barts and the London NHS Trust, Whitechapel, London El 1BB, UK

Funding: Nil.

Conflict of interest: None.

Correspondence to: $M$ Hourihan, Ambrose King Centre, Barts and the London NHS Trust, Whitechapel, London E1 1BB, UK; martin.hourihan@ bartsandthelondon.nhs.uk

Accepted for publication 6 August 2004

\section{REFERENCES}

1 CDSC. Syphilis in Bristol 1997-8: an update. Commun Dis CDR Wkly. (serial online) 1998 (cited 31 July 2003);8:news. Available at www.phls.org.uk/ publications/cdr/CDR98/cdr4798.pdf.

2 Doherty L, Fenton KA, Jones J, et al. Syphilis: old problem, new strategy. BMJ 2002;325: 153 .

3 CDSC. Increased transmission of syphilis in Manchester. Commun Dis Rep CDR Wkly 2000;10:89.

4 Lacey HB, Higgins, Graham D. An outbreak of early syphilis: cases from North Manchester General Hospital. Sex Transm Infect 2001;77:311-13.

5 CDSC. Increased transmission of syphilis in men who have sex with men reported from Brighton and Hove. Commun Dis Rep CDR Wkly 2000;10:177-80.

6 CDSC. Outbreak of heterosexually acquired syphilis in Cambridgeshire. Commun Dis Rep CDR Wkly 2000;10:401-4.

7 Rompalo AM, Lawlor J, Seaman P, et al. Modification of syphilitic genital ulcer manifestations by co-existent HIV infection. Sex Transm Dis 2001;28:448-54.

8 Czelusta A, Yen-Moore A, Van der Straten M, et al. An overview of sexually transmitted diseases. Part III. Sexually transmitted diseases in HIV-infected patients. J Am Acad Dermatol 2000;43:409-32.

9 Goh BT, Van Voorst Vader PC. European guideline for the management of syphilis. Int J STD AIDS 12(Suppl 3):14-26.

10 Chapel TA, The signs and symptoms of secondary syphilis. Sex Transm Dis 1980;7:151-4.

11 Wheeler H, Agarwal S, Goh BT. Dark ground microscopy and treponemal serology tests in the diagnosis of early syphilis. Sex Transm Infect (in press). 
12 Young $\mathbf{H}$, Moyes A, Seagar L, et al. Novel recombinant-antigen enzyme immuno-assay for serological diagnosis of syphilis. J Clin Microbiol 1998;36:913-17.

13 Egglestone SI, Turner AJL for the PHLS syphilis serology working group. Serological diagnosis of syphilis. Commun Dis and Public Health 2000;3:158-62.

14 Schmidt B, Edjlalipour M, Luger A. Comparative evaluation of nine enzymelinked immunosorbant assays for determination of antibodies against Treponema pallidum in patients with primary syphilis. J Clin Microbiol 2000;38: 1279-82.

15 Sadiq ST, Taylor S, Kaye S, et al. The effect of antiretroviral therapy on HIV-1 RNA loads in seminal fluids in HIV-positive patients with and without urethritis. AIDS 2002; 16:219-25.

16 British Medical Association. Medical Foundation for AIDS and Sexual Health. London: BMA, 2003.

17 Winston A, Hawkin D, Mandalia S, et al. Is increased surveillance for asymptomatic syphilis in an HIV outpatient department worthwhile? Sex Transm Infect 2003;79:257-9.
18 Hopkins S, Lyons F, Mulcahy F, et al. The great pretender returns to Dublin Ireland. Sex Trans Infect 2001;77:316-8.

19 Poulton M, Dean GL, Williams DJ, et al. Surfing with spirochaetes: an on going syphilis outbreak in Brighton. Sex Transm Infect 2001:77:319-21.

20 CDSC. Sexually transmitted infections quarterly report: syphilis in England, Wales and Northern Ireland. Commun Dis CDR Wkly (serial online) 2002 (cited 31 October 2003). Available at www.hpa.org.uk/cdr/PDFfiles/2002/ cdr4402.pdf.

21 Lambert N, Phillips A, Imrie J, et al. Community based syphilis screeningacceptable and feasible but ineffective in case finding. Sex Transm Dis 2003;79(Suppl 1):A3.

22 Baguley S, Maple C, Stephenson L, et al. Development of an oral fluid test for treponemal infection. Sex Transm Dis 2003;79(Suppl 1):A28.

23 Rosanna W, Peeling, Htun Ye. Diagnostic tools for preventing and managing maternal and congenital syphilis: an overview. Bull World Health Organ 2004;82:439-46

24 Elford J, Bolding G, Davis $M$, et al. Internet and HIV study: Online survey 2002 preliminary findings. Available at www.city.ac.uk/barts/hsi. 